\title{
La hiperosmolaridad del film lagrimal en el ojo seco
}

\author{
Tears osmolarity in dry eye
}

Alejandro J. Aguilar

Jefe del Departamento de Superficie Ocular Grupo Médico Las Lomas. San Isidro, Buenos Aires- Argentina; Profesor Asociado Cátedra de Oftalmología Universidad del Salvador.

Dirección para correspondencia: Italia 2178 - PB7

(1640) Martinez-Buenos Aires - Argentina

E-mail: aguilara@arnet.com.ar

\section{RESUMEN}

El ojo seco es una compleja enfermedad multifactorial de la película lagrimal y de la superficie ocular caracterizada por síntomas de desconforto, alteraciones de la visión e inestabilidad del film lagrimal que puede provocar daño potencial sobre la superficie ocular. La inestabilidad del film producirá aumentos en la osmolaridad de la película lagrimal, lo que desencadena a su vez lesiones osmóticas epiteliales e inflamación. Al producirse estos cambios sobre la superficie ocular, los mecanismos neurofisiológicos de homeostasis se verán alterados lo que complicará aún más el proceso, con la aparición de circuitos viciosos fisiopatológicos.

Descriptores: Síndromes de ojo seco; Concentración osmolar; Lágrimas/ química

\section{INTRODUCTION}

La superficie ocular es una delicada porción de la anatomía del ojo, donde sus componentes constitutivos guardan una estrecha relación entre sí para lograr mantener la homeostasis de la región, lo que sin lugar a dudas establece la presencia de una verdadera unidad anatomofuncional ${ }^{(1)}$ en la cuál el film lagrimal debe mantener íntegra la salud de los epitelios de la conjuntiva y de la córnea, y a la vez contribuir a la fisiología normal del estroma.

Ahora bien, para que la película lagrimal pueda cumplimentar eficazmente esta función, sus tres capas deben estar íntegras y en constante equilibrio. Las tres capas del film guardan una estrecha relación entre sí, al punto que cualquier alteración que se presente en una de ellas (composicional, secretoria, etc.) puede incidir drásticamente en el equilibrio normal de todas, provocando de esta manera la alteración parcial o total de la película lagrimal y consecuentemente a esto, la alteración de los epitelios.

El ojo seco, es un proceso patológico multifactorial de la superficie ocular producido por una deficiencia en la cantidad y/o en la calidad del film lagrimal que, de esta manera, se hace incapaz de mantener la salud de los epitelios de la córnea y de la conjuntiva. Esto produce metaplasia epitelial de tipo escamosa y daño epitelial ${ }^{(2)}$.

Si bien esta falla puede obedecer a diferentes situaciones: aumento de la evaporación, deficiencia en la producción, alteraciones composicionales; en todos los casos el correlato fisiopatológico es el aumento de la osmolaridad del film ${ }^{(3)}$ que aparece dentro de las primeras 24 horas de iniciado el proceso.

La disminución en la producción de lágrimas y/o cambios cualitativos composicionales, así como el aumento de la evaporación de la película lagrimal favorecen el fenómeno de hiperosmolaridad. La evaporación de un menor volumen para igual superficie hace aumentar la osmolaridad durante las primeras 24 horas de iniciada la disminución volumétrica ${ }^{(4)}$. 
La hiperosomolaridad ${ }^{(5)}$ causa lesión epitelial en forma directa al producir descamación celular, desaparición completa de capas de células epitelialaes superficiales, disminución de la densidad citoplasmática y acumulación de hileras de mucus producto de células mucosecretantes alteradas osmóticamente. Este fenómeno se evidencia generalmente entre los 15 y 30 días del cambio osmolar de la película lagrimal.

Según Holly, Lamberts ${ }^{(6)}$ la formación del film lagrimal es esencialmente un fenómeno de "mojabilidad". El epitelio de la córnea y de la conjuntiva debe estar completamente humidificado por la capa acuosa del film. Para una mojabilidad completa, las condiciones de la superficie ocular necesitan que la tensión superficial de la capa acuosa en la interfase con el epitelio sea menor a la tensión superficial del epitelio expuesto al medio.

Los mucopolisacáridos de la capa mucosa son los principales responsables de mantener una tensión superficial estable. Al producirse acúmulos mucosos y destrucción de células mucosecretantes por aumento de la osmolaridad del film, la tensión superficial se eleva y por lo tanto se inhibe la mojabilidad del epitelio.

En 1993 postulé una hipótesis ${ }^{(7)}$ basada en el fenómeno de osmosis. El principio de osmosis se caracteriza por la presencia de un flujo solvente a través de una membrana semipermeable, que se genera cuando la concentración de la solución aumenta a uno de los dados de la membrana. Este movimiento acuoso tiende a igualar las concentraciones en ambos $\operatorname{lados}^{(8)}$. Cuando esto sucede, el fenómeno osmótico se detiene.

El epitelio córneoconjuntival y la capa mucosa del film constituyen una perfecta membrana biológica semipermeable y por lo tanto se comporta como tal. Al aumentar la osmolaridad de la capa acuosa, el fenómeno osmótico se pone en marcha produciendo un flujo solvente desde los epitelios y la capa mucosa hacia la capa acuosa. Este flujo, alimentado por la presión osmótica, genera una importante fuerza que separa la capa acuosa impidiendo la mojabilidad.

Al mismo tiempo, la deshidratación producida en la capa mucosa generará destrucción del mucus que eleva más la tensión superficial potenciando el desequilibrio osmolar.

En este punto, la lesión sicca se ha producido, se exacerba con la deshidratación celular de las capas celulares del epitelio generada en el proceso anteriormente descripto y entra en un circuito vicioso fisopatológico.

Por otro lado, si tenemos en cuenta la presencia del gradiente acuoso a través de los canales proteicos de agua presentes en el estroma y con dirección hacia el humor acuoso; veremos que una nueva fuerza física de dirección opuesta (fuerza osmótica) podría modificar este movimiento. Este cambio direccional de fluidos producido por la hiperosmolaridad y los mecanismos que la misma produce, puede provocar deshidratación de los proteoglicanos sulfatados (GAGS) que ocupan los espacios entre las fibras de colágeno del estroma $^{(9-10)}$. Al deshidratarse estas estructuras glicoproteicas, el correcto balance hídrico del estroma se verá afectado, lo que incidirá en la mantención normal de la transparencia de la córnea. Al mismo tiempo, la alteración del estroma producirá pérdida del número de células mucosecretantes, con la consiguiente alteración mucosa y tensional ya descripta ${ }^{(11)}$.

De esta manera, la hiperosmolaridad desencadena una serie de fenómenos fisiopatológicos con evidentes efectos de retroalimentación entre ellos, que en uno y otro sentido se potencian entre sí.

No escapa a este análisis que el aumento de la osmolaridad del film lagrimal en el ojo seco, como condición de stress sobre la superficie ocular, dispara el proceso de inflamación y fenómenos inmunológicos como la presentación de autoantígenos que potencian el proceso inflamatorio.

Estudios de marcadores inflamatorios como el NF- $\kappa \beta$ que migra del núcleo al citoplasma en el proceso inflamatorio, están directamente relacionados con el fenómeno de hiperosmolaridad del film lagrimal. La translocación nuclear del NF-к $\beta$ es directamente proporcional al aumento de la osmolaridad de la película lagrimal.

Berra A, Berra $\mathrm{M}^{(12)}$ compararon el proceso de translocación nuclear NF- $\kappa \beta$ en pacientes sanos, en mujeres posmenopáusicas y en pacientes portadores de síndrome de Sjögren y los relacionaron con la osmolaridad del film lagrimal y con la citología de impresión conjuntival de estos pacientes. Los pacientes sanos, con valores de osmolaridad normal (300 mOsm/L +/-10) no evidenciaron la presencia del marcador NF- $\kappa \beta$ ni mostraron cambios metaplásicos conjuntivales (citologías normales grado 0). Las mujeres posmenopáusicas portadoras de ojo seco moderado mostraron aumentos moderados de la osmolaridad del film lagrimal (300-400 mOsm/L), moderada presencia del factor NF- $\kappa \beta$ (entre el $25-70 \%$ de expresión) y cambios metaplásicos celulares grados I-II. Por el contrario, pacientes portadores de ojo seco severo, el grupo síndrome de Sjögren, evidenció altos valores de osmolaridad del film $(<400 \mathrm{mOsm} / \mathrm{L})$, gran expresión de translocación nuclear del factor NF- $\kappa \beta(10-$ $75 \%$ de expresión) y metaplasia escamosa severa (grado II-III).

Khanal et $\mathrm{al}^{(13)}$ compararon los valores de la osmolaridad del film lagrimal en pacientes sanos versus pacientes portadores de ojo seco, demostrando hiperosmolaridad en pacientes con ojo seco y postularon a la medición de la osmolaridad del film como uno de los pilares diagnósticos de ojo seco. De la misma manera, la osmolaridad es una de las pruebas diagnósticas recomendadas por el comité del Instituto Nacional del Ojo de los Estados Unidos de Norteamérica (National Eye Institue) $^{(14)}$.

Estudios de laboratorio permitieron demostrar que tan solo un aumento del $1 \%$ en la osmolaridad del film es capaz de provocar lesiones epiteliales y alterar el flujo normal de líquidos hacia el estroma.

Labbé et al ${ }^{(15)}$ establecieron que el ojo seco es una entidad clinicopatológica que involucra al film lagrimal, a las glándulas lagrimales, a los párpados y que produce un amplio espectro de cambios fisiopatológicos donde la hiperosmolaridad es uno de los principales factores, asignándole un rol diagnóstico preponderante.

Numerosos autores ${ }^{(16-17)}$ confirman estos reportes. 
Si bien el examen de la osmolaridad del film requiere de equipamiento sofisticado y un alto grado de capacitación para realizarlo, podemos evaluar indirectamente su valor a través de la medición de la concentración de sodio del film usando las tiras del papel de Schirmer ${ }^{(7)}$. Siguiendo el método del test del sudor, que consiste en medir la concentración de sodio a través de la utilización de un papel de filtro sobre la epidermis de niños afectados de enfermedad fibroquística del páncreas, evaluamos la concentración de sodio que obtenemos de las lágrimas por humidificación de la bandeleta de papel Wathman 41 de la forma habitual para el test de Schirmer. Posteriormente por medio de un colorímetro (aparato común a todo laboratorio hospitalario), medimos la concentración de sodio presente en el mismo. Normalmente la concentración promedio de sodio presente en el film lagrimal es del órden de los 134-170 meq/L; en pacientes portadores de ojo seco, esta concentración asciende hasta valores extremos $(500 \mathrm{meq} / \mathrm{L})$. Posteriormente, y a través del empleo de la fórmula Van't Hoff, podemos evaluar la presión osmótica del ión sodio, índice indirecto de la osmolaridad del film.

Fórmula Van't Hoff: Presión osmótica $=\mathrm{C} \times \mathrm{R} \times \mathrm{T}$

Donde $\mathrm{C}$ es la concentración de la solución, $\mathrm{R}$, la constante universal de los gases y $\mathrm{T}$ la temperatura absoluta.

Sin lugar a dudas, el ojo seco constituye en la actualidad uno de los problemas más comunmente diagnosticados por los oftalmólogos. El conocimiento de su cascada fisiopatológica y su diagnóstico precoz, nos permitirá un mejor manejo de esta patología. En este sentido, el evaluar la osmolaridad del film en estos pacientes, si bien no nos brinda un diagnóstico etiológico de la enfermedad, nos proporciona una eficaz herramienta de diagnóstico y evaluación de la enfermedad, ya que sus valores son directamente proporcionales con la severidad del cuadro clínico de ojo seco, y esta siempre presente en estos pacientes.

\section{ABSTRACT}

Dry eye is a complex multifactorial disease of the tears and ocular surface that results in symptoms of discomfort, visual disturbance, and tear film instability with potential damage to the ocular surface. It is accompanied by increased osmolarity of the tear film and inflammation of the ocular surface.
Inestability of the tear film may also cause an increased osmolarity of the tear film, thus triggering epithelial osmotic lesions and inflammation. The occurrence of such changes on the eye surface results in disturbances of homeostatic neurophysiologic mechanisms which further worsen the process and vicious pathophysiological cycles occur.

Keywords: Dry eye syndromes; Osmolar concentration; Tears/chemistry

\section{REFERENCIAS}

1. Aguilar AJ. Ojo seco: manual sobre fisiopatogenia, diagnóstico y tratamiento. Buenos Aires: Ediciones Científicas Argentinas; 1999. p.196.

2. Aguilar AJ, Fonseca L, Croxatto OJ. Sjögren's syndrome: a comparative study of impression cytology of the conjunctiva and the buccal mucosa, and salivary gland biopsy. Cornea. 1991;10(3):203-6.

3. Gilbard JP, Farris RL. Tear osmolarity and ocular surface disease in keratoconjunctivitis sicca. Arch Ophthalmol. 1979;97(9):1642-6.

4. Holly FJ, Patten JT, Dohlman CH. Surface activity determination of aqueous tear components in dry eye patients and normals. Exp Eye Res. 1977;24(5): 479-91.

5. Gilbard JP, Farris RL, Santamaria J $2^{\text {nd }}$. Osmolarity of tear microvolumes in keratoconjunctivitis sicca. Arch Ophthalmol. 1978;96(4):677-81.

6. Holly FJ, Lamberts DW. Effect of nonisotonic solutions on tear film osmolarity. Invest Ophthalmol Vis Sci. 1981;20(2):236-45.

7. Aguilar AJ, Rodriguez M, Fonseca L, Marré D. El test de Schiemer como método de medida de la concentración de Sodio del film lagrimal y de su osmolaridad en pacientes portadores de ojo seco: claves de la fisiopatología de la lesión sicca. Arch Oftalmol B Aires. 1984;68:128-38.

8. Glasstone D. Elementos de fisicoquímica. Buenos Aires: Editorial Médico Quirúrgica; 1952. p.847.

9. Fujikawa LS, Foster CS, Gipson IK, Colvin RB. Basement membrane components in ealing rabbit corneal epithelial wounds: immunofluorescence and ultrastructural studies. J Cell Biol. 1984;98(1):128-38.

10. Funderburgh JL, Cintron C, Covington HI, Conrad GW. Inmunoanalysis of keratan sulfate proteoglycan from corneal scars. Invest Ophthalmol Vis Sci. 1988;29(7):1116-24.

11. Tseng SC, Hirst LW, Maumenee AE, Kenyon KR, Sun TT, Green WR. Possible mechanisms for the loss of goblet cells in mucin-deficient disorders. Ophthalmology. 1984;91(6):545-52.

12. Berra A, Berra M. Hyperosmolarity induce nuclear translocation of NF- $\kappa B$ in human conjunctiva epithelial cells. Invest Opthalmol Vis Sci. 2005;46.

13. Khanal S, Tomlinson A, McFadyen A, Diaper C, Ramaesh K. Dry eye diagnosis. Invest Opthalmol Vis Sci. 2008;49(4):1407-14.

14. Lemp MA. Report of the National Eye Institute/Industry workshop on Clinical Trials for Dry Eyes. CLAO J. 1995;21(4):221-32.

15. Labbé A, Brignole-Baudouin F, Baudouin C. Ocular surface Investigations in dry eye. J Fr Ophtalmol. 2007;30(1):76-97.

16. Tomlinson A, Khanal S, Ramaesh K, Diaper C, McFadyen A. Tear film osmolarity: determination of a referent for dry eye diagnosis. Invest Ophthalmol Vis Sci. 2006;47(10):4309-15.

17. Berra I, Aguilar AJ, Berra A. Clinical and laboratory tests in patients with dry eye, allergic conjunctivitis and dry eye plus allergic conjunctivitis. In: ARVO Meeting;1999. Florida; May 9-14. 